\title{
Improved Social Interaction, Recognition and Working Memory with Cannabidiol Treatment in a Prenatal Infection (poly I:C) Rat Model
}

\author{
Ashleigh L Osborne ${ }^{1,2}$, Nadia Solowij ${ }^{3}$, Ilijana Babic ${ }^{1,2,4}$, Xu-Feng Huang ${ }^{1,2}$ and Katrina Weston-Green ${ }^{* 1,2}$ \\ 'Centre for Translational Neuroscience, School of Medicine, Faculty of Science, Medicine and Health, and Illawarra Health and Medical Research \\ Institute, University of Wollongong, Wollongong, NSW, Australia; ${ }^{2}$ Centre for Medical and Molecular Biosciences, Faculty of Science, Medicine and \\ Health, University of Wollongong, Wollongong, NSW, Australia; ${ }^{3}$ School of Psychology, Faculty of Social Sciences and Illawarra Health and Medical \\ Research Institute, University of Wollongong, Wollongong, NSW, Australia; ${ }^{4}$ Illawarra Shoalhaven Local Health District, Wollongong, NSW, Australia
}

\begin{abstract}
Neuropsychiatric disorders such as schizophrenia are associated with cognitive impairment, including learning, memory and attention deficits. Antipsychotic drugs are limited in their efficacy to improve cognition; therefore, new therapeutic agents are required. Cannabidiol (CBD), the non-intoxicating component of cannabis, has anti-inflammatory, neuroprotective and antipsychotic-like properties; however, its ability to improve the cognitive deficits of schizophrenia remains unclear. Using a prenatal infection model, we examined the effect of chronic CBD treatment on cognition and social interaction. Time-mated pregnant Sprague-Dawley rats $(n=16)$ were administered polyinosinic-polycytidilic acid (poly l:C) (POLY; $4 \mathrm{mg} / \mathrm{kg}$ ) or saline (CONT) at gestation day I5. Male offspring (PN56) were injected twice daily with $10 \mathrm{mg} / \mathrm{kg} C B D(C O N T+C B D, P O L Y+C B D ; n=12$ per group) or vehicle $(V E H ; C O N T+V E H, P O L Y+V E H ; n=12$ per group) for 3 weeks. Body weight, food and water intake was measured weekly. The Novel Object Recognition and rewarded T-maze alternation tests assessed recognition and working memory, respectively, and the social interaction test assessed sociability. POLY+VEH offspring exhibited impaired recognition and working memory, and reduced social interaction compared to CONT+VEH offspring ( $p<0.0 \mathrm{I})$. CBD treatment significantly improved recognition, working memory and social interaction deficits in the poly l:C model ( $p<0.01$ vs POLY $+\mathrm{VEH}$ ), did not affect total body weight gain, food or water intake, and had no effect in control animals (all $p>0.05$ ). In conclusion, chronic CBD administration can attenuate the social interaction and cognitive deficits induced by prenatal poly l:C infection. These novel findings present interesting implications for potential use of CBD in treating the cognitive deficits and social withdrawal of schizophrenia.
\end{abstract} Neuropsychopharmacology (2017) 42, I447-|457; doi: I0.I038/npp.2017.40; published online 22 March 2017

\section{INTRODUCTION}

Evidence suggests that exposure to a prenatal infection during the first or second trimester of pregnancy can disrupt neurodevelopment, increasing the risk of developing neuropsychiatric disorders such as schizophrenia (Meyer, 2013). Prenatal infection with polyinosinic-polycytidilic acid (poly I:C), a synthetic double-stranded RNA virus, is a welldocumented rodent model of schizophrenia-like phenotypes (Meyer, 2014; Meyer and Feldon, 2012). Following administration to the pregnant dam, poly I:C binds to the Toll-like receptor 3 and initiates a maternal immune response that raises proinflammatory cytokines in the placenta, amniotic

\footnotetext{
*Correspondence: Dr K Weston-Green, Centre for Translational Neuroscience, School of Medicine, Faculty of Science, Medicine and Health, and Illawarra Health and Medical Research Institute, University of Wollongong, Northfields Avenue, Wollongong, NSW 2522, Australia, Tel: +6I 24252 8506, Fax: +6I 2422 I 315I,

E-mail: katrina_green@uow.edu.au

Received 24 October 2016; revised 10 February 2017; accepted 19 February 2017; accepted article preview online 23 February 2017
}

fluid, and fetal brain (Meyer, 2013; Meyer et al, 2009). Neuro-inflammation in the foetal brain activates microglia, causing white matter injury and neuronal apoptosis (Meyer, 2013). Consequently, behavioral (eg deficits in cognition, social interaction and sensorimotor gating), neurochemical (eg striatal dopaminergic hyperfunction) and structural (eg reduced hippocampal and prefrontal cortical volumes) brain changes are observed in poly I:C offspring, which reflect observations in schizophrenia patients (as reviewed in Meyer, (2014)). These alterations emerge in the offspring during early adulthood, reflecting the delayed onset of schizophrenia symptoms observed in the clinic (Meyer, 2013). Moreover, some of the behavioral and structural changes induced by poly I:C can be reversed with clozapine and risperidone administration (Piontkewitz et al, 2009, 2011). Therefore, the poly I:C model shows construct, face and predictive validity, making it a useful tool to investigate pharmacological interventions for the treatment of schizophrenia (Meyer, 2014).

Cognitive dysfunction, including impairments to memory, attention, and executive functioning, is experienced by 
$75-85 \%$ of people with schizophrenia (Barch and Ceaser, 2012). Cognitive deficits often precede the onset of other symptoms and are associated with poor medication compliance and a higher tendency for relapse in first episode psychosis (Meyer et al, 2011b). In fact, cognitive deficits are thought to be a better prognostic indicator in patients than other symptom domains, as earlier disease onset correlates with the severity of cognitive dysfunction (Gray and Roth, 2007) and cognitive dysfunction predicts clinical course and future functional outcomes (Green, 2006). The negative symptoms, which include alogia (lack of speech), avolition (lack of motivation), anhedonia (inability to feel pleasure), and social withdrawal, are frequently associated with disturbances to psychosocial functioning, relationships, employment and overall quality of life (Lindenmayer et al, 2013). While the positive symptoms of schizophrenia (hallucinations and delusions) are usually controlled by antipsychotic drug (APD) treatment, the drugs have minimal efficacy to improve the cognitive symptoms (Gray and Roth, 2007) and one-third of patients who experience negative symptoms are APD-resistant (Lindenmayer et al, 2013). Furthermore, APDs can cause side effects, particularly motor and metabolic (obesity and type 2 diabetes mellitus) disturbances (Weston-Green et al, 2013). Therefore, there is a requirement to improve the pharmacological treatment of schizophrenia.

A growing body of evidence demonstrates that cannabidiol (CBD), a component of Cannabis sativa, prevents hallucinations and cognitive impairment induced by cannabis and delta-9-tetrahydrocannabinol $(\Delta 9$-THC) administration (reviewed in Schubart et al (2014)). Studies reported that CBD limited the positive and negative schizophrenia-like phenotypes in the MK-801 model, suggesting that CBD has antipsychotic potential (reviewed in Schubart et al (2014)). These findings translate to the clinic, where CBD treatment improved positive and negative symptoms in schizophrenia patients, performing comparably to a current APD, amisulpride, with fewer side effects (Leweke et al, 2012). A number of preclinical models of cognitive impairment suggest that CBD improves learning and memory (as reviewed in Osborne et al (2017)); however, the effect of CBD on the cognitive deficits of schizophrenia is unclear. For example, acute $\mathrm{CBD}$ did not improve selective attention in schizophrenia outpatients (Hallak et al, 2010), or MK-801-induced social recognition memory deficits in rats (Deiana et al, 2015). CBD was protective against MK-801-induced object recognition memory impairment at high doses (Gomes et al, 2015a,b); its effect on other cognitive domains impaired in schizophrenia (ie working memory) is unknown. A disadvantage of pharmacological models is that they do not incorporate neurodevelopmental or genetic approaches, which underlie the aetiology of schizophrenia in humans (Mouri et al, 2013). Therefore, the aim of this study was to determine whether chronic CBD treatment could improve recognition and working memory impairment, and social withdrawal in a neurodevelopmental model of schizophrenia-like phenotypes. Body weight, food and water intake was also measured to determine whether $\mathrm{CBD}$ induces weight gain or hyperphagia.

\section{MATERIALS AND METHODS}

This study is reported in accordance with the Animal Research: Reporting of In Vivo Experiments (ARRIVE) guidelines (Kilkenny et al, 2010). The completed 'ARRIVE Guidelines Checklist' for reporting the animal data in this study are included in the Supplementary Information (Supplementary Figure S1).

\section{Ethics Statement}

Experimental procedures were approved by the Animal Ethics Committee of the University of Wollongong, Wollongong, NSW, Australia (AE15/05) and complied with the Australian Code of Practice for the Care and Use of Animals for Scientific Purposes (National Health and Medical Research Council (NHMRC), 2013). All efforts were made to minimize the number and suffering of animals.

\section{Animals and Drug Treatment}

Sixteen time-mated pregnant (gestation day 8) SpragueDawley rats (12 weeks old) were obtained from the Animal Resources Centre (Perth, WA, Australia), housed at the Animal Research Facility, University of Wollongong and habituated to the new environment for 1 week. Rats were housed individually on corncob bedding with plastic tunnels and nesting material for environmental enrichment, under a light-dark cycle (photophase $1900-0700 \mathrm{~h}$ ) at $22^{\circ} \mathrm{C}$ and allowed ad libitum access to standard laboratory chow (3.9 kcal/g; 10\% fat, $74 \%$ carbohydrate, $16 \%$ protein) and water. On gestation day 15 (GD15; at $0900 \mathrm{~h}$ ), dams received a single intravenous injection $(1 \mathrm{ml} / \mathrm{kg})$ of either poly I:C ( $4 \mathrm{mg} / \mathrm{kg}$, Sigma-Aldrich, Sydney, NSW, Australia; $n=8$ ) or saline (control; $n=8$ ) to the lateral tail vein. The dose, route of administration and timing of poly I:C administration was based on previous studies, in which behavioral impairments observed in the offspring mimic negative/cognitive schizophrenia-like phenotypes (Meyer and Feldon, 2012). Following birth, pups were culled to $\sim 10$ pups per litter and offspring were maintained with their respective dams until weaning on postnatal day 21 (PN21). Offspring were then pair-housed with littermates, with ad libitum access to standard laboratory chow and water. Pairs of male offspring were assigned to receive intraperitoneal (i.p) injections of either CBD (10 mg/kg; THC-Pharm GmbH, Frankfurt, Germany) dissolved in Tween 80 and saline (vehicle (VEH); $1: 16(v / v)$; Sigma-Aldrich), or VEH alone (injection volume of $5 \mathrm{ml} / \mathrm{kg}$ ), with an even spread of litters in each treatment group in order to control for potential litter effects. Drug solutions were prepared immediately prior to each drug administration, which occurred from PN56, equating to late adolescence/early adulthood in humans (Andersen and Navalta, 2004). Treatments were administered for 3 weeks, twice a day, at $12 \mathrm{~h}$ intervals $(0700 \mathrm{~h}, 1900 \mathrm{~h})$, based on the half-life of CBD in the rat brain following i.p. injection (Deiana et al, 2011). The CBD dosage and treatment duration was based on previous studies that reported positive effects of CBD on cognition in inflammation-based rodent models (Barichello et al, 2012; Cassol-Jr et al, 2010; Fagherazzi et al, 2011; Schiavon et al, 2014). The experimental design resulted in four conditions: (1) offspring 
a

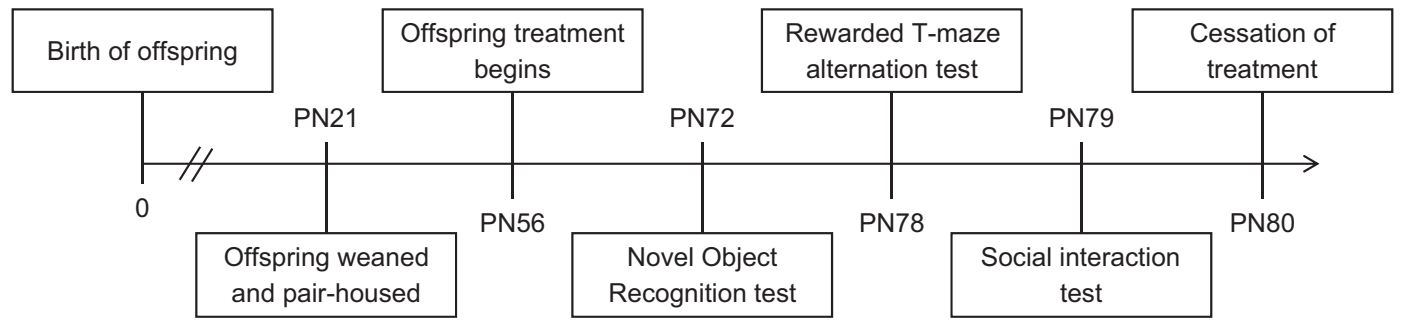

b

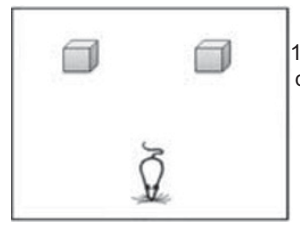

Familiarization trial

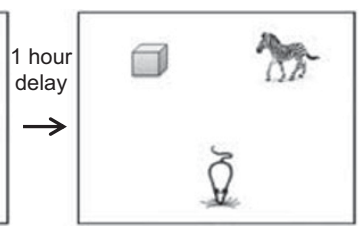

Novel object test

C

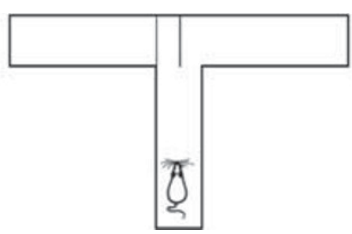

Forced run

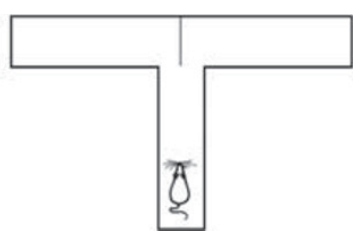

Choice run

Figure I Experimental design and methods used to investigate the effects of cannabidiol (CBD) treatment on offspring behavior in the poly l:C rat model. (a) The experimental timeline used in the present study with the age of offspring in postnatal (PN) days. (b) A schematic of the Novel Object Recognition test used to assess recognition memory of offspring. (c) A schematic of the rewarded T-maze alternation test used to assess working memory performance of offspring.

of control dams administered vehicle (CONT+VEH; $n=12)$; (2) offspring of control dams administered CBD (CONT $+\mathrm{CBD} ; n=12)$; (3) offspring of poly I:C dams administered VEH (POLY+VEH; $n=12$ ); and (4) offspring of poly I:C dams administered CBD (POLY+CBD; $n=12$ ). Body weight, food and water intake (per cage ie 2 rats) were recorded weekly during the 3 -week treatment period.

\section{Behavioral Testing}

After 2 weeks of $\mathrm{CBD}$ or $\mathrm{VEH}$ treatment, rat offspring underwent a series of behavioral tests to examine social interaction, learning and memory (Figure 1a). Behavioral tests were conducted during the active phase (0900 and $1800 \mathrm{~h}$ ) with a 24 -h rest period between tests to minimize stress. The time of daily testing was counterbalanced across the experimental groups. Rat behavior was recorded using standard commercial cameras (Logitech Pty, Alexandria, Australia) and de-identified video recordings were later analysed. Equipment was cleaned with $70 \%$ ethanol between trials to eliminate olfactory cues. The behavioral tests were performed in order of invasiveness as described below.

Novel object recognition (NOR) test. The NOR test was performed as described previously, with minor modifications (Bevins and Besheer, 2006). Two objects (plastic building blocks) were placed in the home cages for 24-h familiarization. In the first trial (familiarization), a rat was placed in an arena (black square arena $60 \mathrm{~cm} \times 60 \mathrm{~cm} \times 60 \mathrm{~cm}$, with even lighting of 20 lux) to habituate for 5 min then returned to its home cage. The two familiar objects were placed in opposite corners of the upper half of the arena and the rat was positioned in the centre of the far wall (ie the lower half of the arena), facing away from the objects (Figure 1b). The rat was left to explore the arena for $5 \mathrm{~min}$ then returned to its home cage for $1 \mathrm{~h}$. In the second trial, one of the familiar objects was replaced with a novel object (toy figurine) in the arena and the rat was allowed to explore for $3 \mathrm{~min}$. Time spent interacting with the objects was recorded, with interaction defined as direct contact with the object, including touching an object with the mouth, nose or paw. Accidental contact (ie bumping into the object in passing) and contact with the object as a means to explore other aspects of the arena (ie rearing) were not scored as object interaction (Bevins and Besheer, 2006). A discrimination ratio was calculated for each rat expressed as: $T_{\mathrm{N}} /\left(T_{\mathrm{F}}+T_{\mathrm{N}}\right)$, where $T_{\mathrm{N}}=$ time spent exploring the novel object (s) and $T_{\mathrm{F}}=$ time spent exploring the familiar object (s). Values for the discrimination ratio ranged from 0 to 1 , where a score closer to 1 indicated greater preference for the novel object, while a score closer to 0 indicated preference for the familiar object.

Rewarded T-maze alternation test. Working memory was assessed using the T-maze rewarded alternation method described previously (Deacon and Rawlins, 2006). The apparatus consisted of a black, T-shaped maze $(50 \mathrm{~cm}$ long $\times 10 \mathrm{~cm}$ wide, $30 \mathrm{~cm}$ high walls with even lighting of 20 lux), containing removable dividers to control access into the left and right arms. A central partition was used to limit access to only one arm of the T-maze at a time and increase alternation rate (Deacon and Rawlins, 2006) (Figure 1c). Prior to testing, rats were acclimatized to a reward stimulus (chocolate pellets) in their home cages. Food was restricted to $5 \mathrm{~g}$ per $100 \mathrm{~g}$ body weight overnight prior to the habituation, training, and testing days. In the habituation trial, the chocolate pellet rewards were placed at the end of the left and right arms of the T-maze and the dividers were removed. A rat was placed at the start arm and allowed to freely explore the maze for $5 \mathrm{~min}$. After the habituation trial, the rat was returned to its home cage. Habituation was considered successful when rats consumed reward pellets from both arms of the T-maze (Deacon and Rawlins, 2006). As rats completed the habituation trial successfully, no 
further habituation trials were required. Rats then underwent training where they were taught to alternate entry into the left and right arms of the T-maze using (1) a 'forced' run: one arm of the T-maze was closed by a divider and the reward stimulus placed in the open arm, and (2) a 'choice' run: divider was removed opening both arms of the T-maze and the reward stimulus was positioned in the newly opened arm. Training took place over three consecutive training days and consisted of eight trials (four forced and four choice runs) per day, with randomized alternation of the divider position for each forced run. Training was considered successful when control rats achieved an $80 \%$ trial accuracy, as described by Deacon and Rawlins (2006). On the test day, the same alternation procedures were conducted as described above, with a total of 10 trials (5 forced runs, 5 choice runs) per rat. The time delay between the forced and choice runs was limited to $30 \mathrm{~s}$ and the total trial time was $3 \mathrm{~min}$. A correct response was accepted as first entry into the correct arm. Trials were excluded from analysis if the rat jumped out of the maze. The percentage of correct responses and the latency to correct entry (s) were recorded for each rat.

Social interaction test. The social interaction test was used to assess the effects of CBD on sociability based on methods previously described by our laboratory (De Santis et al, 2016; $\mathrm{Du}$ Bois et al, 2008). This test capitalizes on the natural tendency of rats to interact with each other; therefore, a lack of interaction can be considered as social withdrawal, reflecting the negative symptoms of schizophrenia (Wilson and Koenig, 2014). Two rats from the same treatment group, which had not been exposed to each other previously (ie unfamiliar), were placed in opposite corners of a black square arena (as described in the NOR methods). Rats were allowed to freely roam the arena for $7 \mathrm{~min}$ and were then returned to their home cage. The amount of time that the rats spent interacting with each other (defined as time spent sniffing, following, grooming, climbing each other) was recorded (s) for each pair of rats. Interaction times for each pair were then collated for statistical analysis and the average interaction time per treatment group was calculated.

\section{Statistical Analysis}

All statistical analyses were conducted using SPSS (Version 21.0, IBM, Illinois, USA). The data were tested for normality using Shapiro Wilk tests. The data points that were $2 \mathrm{SD}$ outside the mean were considered as outliers and removed from further analysis. A two-way ANOVA was used to analyse normally distributed mean total body weight gain, accumulated food and water intake, NOR, and the social interaction test data for main effects of 'prenatal infection' and 'offspring treatment'. Where significant interactions were observed, pairwise comparisons between groups were made with Bonferonni's adjustment for multiple comparisons. The T-maze data remained non-normally distributed following log transformation; the data were therefore analysed using Mann-Whitney $U$ tests for comparisons between groups, with Bonferroni's correction. Comparisons were made between groups to assess the impact of prenatal infection (CONT+VEH vs POLY+VEH), the efficacy of CBD treatment in the model (POLY+VEH vs POLY+CBD), the effect of $\mathrm{CBD}$ administration in control offspring (CONT $+\mathrm{VEH}$ vs $\mathrm{CONT}+\mathrm{CBD}$ ) and to determine whether $\mathrm{CBD}$ treatment restored behavior in the poly I:C model to controllike levels (POLY+CBD vs CONT+VEH). One-sample $t$-tests were used to determine whether NOR performance and percentage of correct entries on the T-maze test, were above chance levels (50\%). Significance was set to $p<0.05$.

\section{RESULTS}

\section{Novel Object Recognition Test}

The discrimination ratio data revealed a significant interaction between 'prenatal infection' and 'offspring treatment' $\left(F_{(1,37)}=10.39, p=0.003\right)$. POLY+VEH offspring had a lower discrimination ratio than $\mathrm{CONT}+\mathrm{VEH}$ offspring $(p=0.001)$ (Figure 2a), indicating a recognition memory deficit following maternal poly I:C exposure. CBD treatment significantly improved discrimination ratio scores of poly I:C offspring (POLY+CBD vs POLY+VEH, $p=0.003$ ) (Figure 2a). There was no significant difference between the discrimination ratios of $\mathrm{VEH}$ and $\mathrm{CBD}$-treated control rats
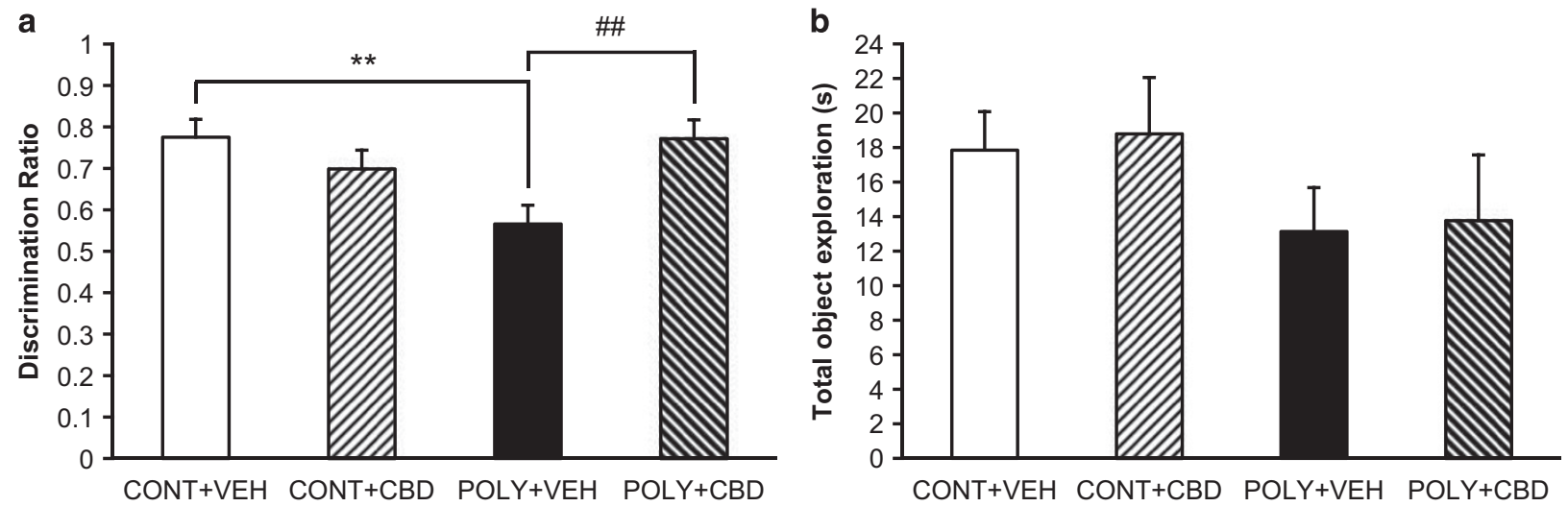

Figure 2 The effect of cannabidiol (CBD) or vehicle (VEH) administration on recognition memory of control (CONT) and poly I:C (POLY) offspring in the Novel Object Recognition test. (a) Maternal poly l:C exposure significantly reduced discrimination ratio scores compared to control male offspring, which was attenuated by CBD treatment ( $n=10$ rats per group). (b) All groups showed comparable total object exploration times $(n=9-1 \mid$ rats per group). The data are presented as mean \pm SEM. ${ }^{*} * p<0.01$ vs CONT+VEH rats, ${ }^{\#} p<0.01$ vs POLY +VEH rats. 


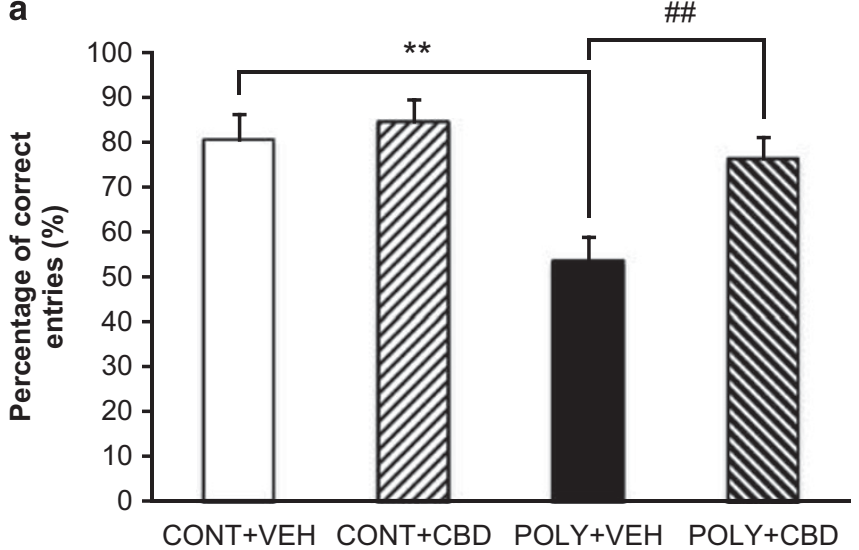

b

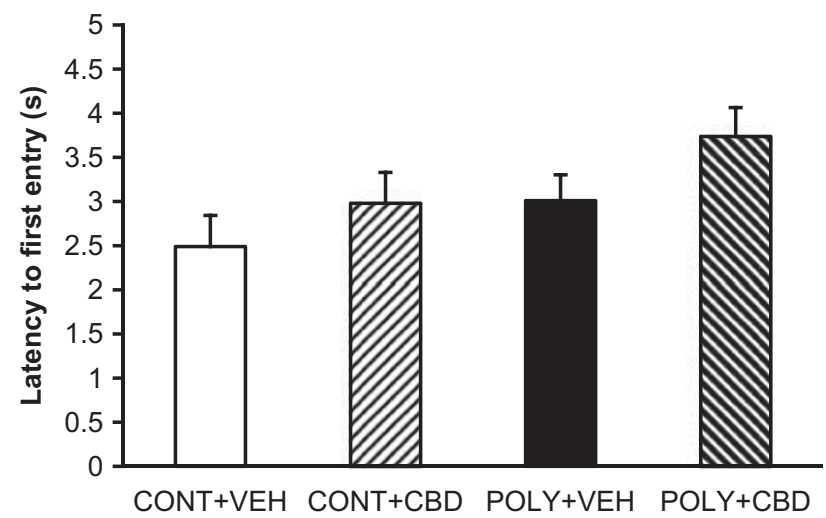

Figure 3 The effect of cannabidiol (CBD) or vehicle (VEH) administration on working memory of control (CONT) and poly I:C (POLY) offspring in the rewarded T-maze alternation test. (a) CBD treatment attenuated poly l:C-induced working memory deficits in male offspring ( $n=10-12$ rats per group). (b) The latency to first entry did not differ between treatment groups $(n=10-12$ rats per group). The data are presented as mean \pm SEM. $* * *<0.01$ compared to CONT+VEH rats, ${ }^{\# \#} p<0.01$ compared to POLY+VEH rats.

(CONT+VEH vs CONT+CBD, $p=0.205)$, indicating that $\mathrm{CBD}$ administration had no effect on recognition memory in healthy rats (Figure 2a). In fact, all groups except the POLY $+\mathrm{VEH}$ group showed a significant preference for the novel object that was above chance levels (CONT+VEH, $t(9)=12.03, p<0.001 ; \mathrm{CONT}+\mathrm{CBD}, t(9)=4.90, p=0.001$; POLY+VEH, $t(9)=1.37, p=0.205$; POLY+CBD, $t(9)=5.23$, $p=0.001 ; n=10$ per group). There was no effect of 'prenatal infection' $\left.\left(F_{(1,36}\right)=2.729, p=0.107\right)$ or 'offspring treatment' $\left.\left(F_{(1,36}\right)=0.072, p=0.790\right)$, nor an interaction between the two factors $\left(F_{(1,36)}=0.003, p=0.958\right)$ on total object exploration time (Figure $2 \mathrm{~b}$ ).

\section{Rewarded T-Maze Alternation Test}

Offspring exposed to prenatal infection had a significantly lower percentage of correct entries compared to control counterparts (POLY+VEH vs CONT+VEH, $p=0.005$ ) (Figure 3a), indicating that exposure to prenatal infection impaired aspects of working memory. CBD treatment attenuated the working memory deficits in poly $\mathrm{I}$ : $\mathrm{C}$ offspring $(\mathrm{POLY}+\mathrm{VEH} v s \mathrm{POLY}+\mathrm{CBD}, p=0.009)$ (Figure 3a), restoring T-maze performance to control-like levels (POLY+CBD vs CONT+VEH, $p=0.561)$. CBD administration did not affect the working memory performance of control offspring (CONT+VEH vs CONT+CBD, $p=0.686)$. When the percentage of correct entries was compared to chance, all groups except the POLY+VEH group preferred to enter the correct $\operatorname{arm}(\mathrm{CONT}+\mathrm{VEH}, t(9)=5.40, p<0.001, n=10 ; \mathrm{CONT}$ $+\mathrm{CBD}, \quad t(10)=7.08, \quad p<0.001, \quad n=11 ; \quad \mathrm{POLY}+\mathrm{VEH}$, $t(11)=0.69, \quad p=0.503, n=12 ; \quad$ POLY + CBD,$\quad t(10)=5.64$, $p<0.001 ; n=11)$. Additionally, there were no significant differences between treatment groups for the latency to first entry in the T-maze test (all $p>0.05$ ) (Figure $3 \mathrm{~b}$ ).

\section{Social Interaction Test}

A two-way ANOVA revealed a significant effect of 'prenatal infection' $\left(F_{(1,18)}=9.11, p=0.007\right)$ and 'offspring treatment' $\left(F_{(1,18)}=10.14, p=0.005\right)$ on total interaction time and a significant interaction between the two factors $\left(F_{(1,18)}=9.21\right.$, $p=0.007)$. Further investigation showed that POLY $+\mathrm{VEH}$

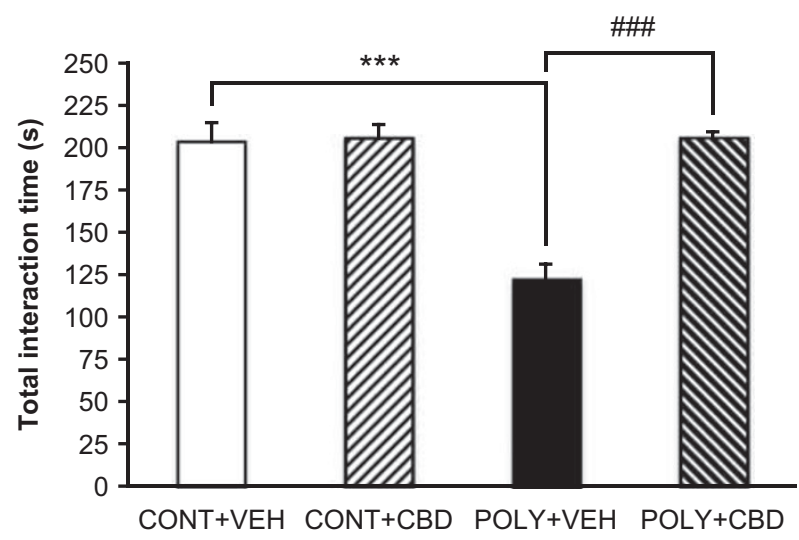

Figure 4 Effect of cannabidiol (CBD) or vehicle (VEH) administration on mean total interaction time of control (CONT) and poly l:C (POLY) offspring in the social interaction test. The data are presented as mean \pm SEM, for pairs of rats ( $n=5$ pairs per control group; $n=6$ pairs/ poly I:C group). ${ }^{*} * * * 0<0.00$ I vs CONT+VEH rats, ${ }^{\# \#} p<0.00$ I vs POLY $+\mathrm{VEH}$ rats.

offspring spent significantly less time interacting compared to their CONT+VEH counterparts $(p<0.001)$, indicating a social interaction deficit resulting from maternal poly I:C exposure (Figure 4). CBD treatment significantly increased total interaction time (POLY+CBD vs POLY+VEH, $p<0.001 ; n=6$ pairs/group) (Figure 4). CBD treatment did not alter the social behavior of control offspring (CONT $+\mathrm{VEH} v s \mathrm{CONT}+\mathrm{CBD}, p=0.921 ; n=5$ pairs per group) (Figure 4).

\section{Body Weight, Food and Water Intake}

There were no interactions between 'prenatal infection' or 'offspring treatment' on mean total body weight gain $\left(F_{(1,43)}\right.$ $=0.37, p=0.549$ ) (Figure 5a), accumulated food intake $\left(F_{(1,20)}=0.21, p=0.651\right)$ (Figure $\left.5 \mathrm{~b}\right)$ or accumulated water intake $\left(F_{(1,20)}=0.001, p=0.970\right)$ (Figure 5c), indicating that poly I:C exposure and CBD administration did not alter basic metabolic parameters in this study. 

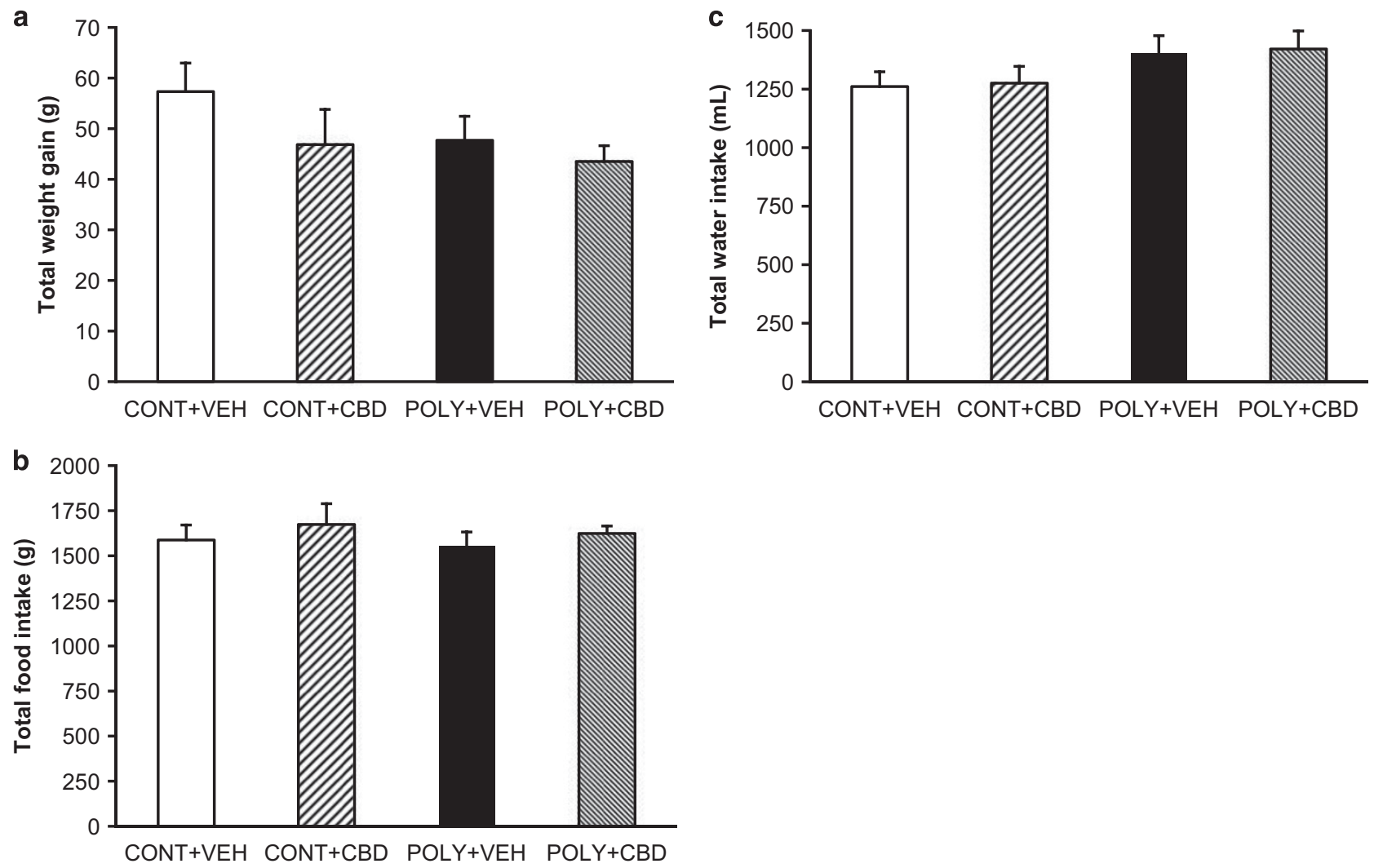

Figure 5 The effect of cannabidiol (CBD; $10 \mathrm{mg} / \mathrm{kg})$ or vehicle $(\mathrm{VEH})$ administration on basic metabolic parameters in control (CONT) and poly I:C (POLY) male offspring. (a) Maternal poly I:C exposure and CBD treatment had no effect on mean total body weight gain. (b) All groups showed comparable mean accumulated food and (c) water intake over the course of the study. The data are presented as mean \pm SEM, $n=12$ rats per group (except the CONT $+\mathrm{CBD}$ group, where $n=1$ I) for body weight gain and $n=6$ pairs of rats per group for mean accumulated food and water intake.

\section{DISCUSSION}

To our knowledge, this is the first study to investigate the effect of chronic CBD treatment $(10 \mathrm{mg} / \mathrm{kg}$, twice-daily) on social interaction and cognitive deficits of adult male offspring exposed to prenatal infection. For the first time, this study has shown that CBD treatment restores working memory deficits in a model of schizophrenia-like phenotypes. This suggests that chronic CBD treatment may be beneficial in the treatment of cognitive and social interaction deficits associated with neuropsychiatric disorders in the clinical scenario. The animal model used in the present study is a well-described neurodevelopmental model that mimics aspects of the behavioral, chemical, and structural brain alterations reported in schizophrenia patients (reviewed in Meyer and Feldon (2012)). Prenatal poly I:C infection has also been used to model autism, which shares similar aetiology and some overlapping symptom domains with schizophrenia. Modeling of autism phenotypes primarily involves poly I:C administration to rodents and non-human primates during early to mid-gestation to elicit repetitive behavior (measured in the marble-burying test), motor stereotypy (such as increased self-grooming), and decreased sociability in offspring (reviewed in Careaga et al (2016)). In the present study, poly I:C was administered during mid-tolate gestation (GD15), which induces decreased sociability in offspring, and has been shown to model other negative and cognitive behavioral phenotypes relevant to schizophrenia
(Meyer and Feldon, 2012). In addition, evidence demonstrates delayed onset of symptoms until early adulthood in these offspring (reviewed in Meyer (2014), which closely resembles schizophrenia rather than autism (Meyer et al, 2011a).

Social interaction test results confirmed the presence of a negative phenotype in male rat offspring exposed to poly I:C during mid-to-late gestation. Previous studies reporting social interaction deficits vary in the dose and gestational timing of poly I:C administration, as well as the social interaction test protocol used (Buschert et al, 2016; Ibi et al, 2009; Labouesse et al, 2015; Ratnayake et al, 2014; Richetto et al, 2015; Zhu et al, 2014). Overall, our study adds to the growing body of literature, suggesting that social interaction deficits are a robust phenotype of the poly I:C model, regardless of the species, strain, dose, and gestational timing of poly I:C administration. In the present study, CBD treatment rescued social interaction in poly I:C offspring, indicating an antipsychotic potential for CBD. This result coincides with a clinical trial where CBD significantly reduced negative symptoms (as assessed by the Positive and Negative Syndrome Scale) in schizophrenia patients after 2 weeks and continued to lower scores until the end of treatment (4 weeks) (Leweke et al, 2012). On the contrary, a recent study investigated the effect of $\mathrm{CBD}$ on social interaction in the poly I:C model and reported no effect of low-dose CBD treatment during peri-adolescence $(1 \mathrm{mg} / \mathrm{kg}$; from PN30 to PN60) on social interaction of mice 
(Peres et al, 2016). Importantly, that study also failed to model social interaction deficits in poly I:C offspring, possibly due to the low dose and gestational timing of poly I:C exposure (10 mg/kg on GD9) (Peres et al, 2016). Mid-tolate gestational poly I:C exposure is typically used to model negative behavioral phenotypes (Meyer and Feldon, 2012); however, early gestational exposure (GD9) can elicit social interaction deficits in mice (Buschert et al, 2016), but may require double the dose $(20 \mathrm{mg} / \mathrm{kg})$ that was used by Peres et al (2016). Previous studies modeling negative behavioral phenotypes have reported a beneficial effect of CBD on social interaction in the MK-801 model (Gomes et al, 2015a,b; Gururajan et al, 2011, 2012; Long et al, 2012) with no effect in spontaneously hypertensive rats (strain that presents with hyperlocomotion and social withdrawal phenotypes), while low-dose CBD ( $1 \mathrm{mg} / \mathrm{kg})$ enhanced social interaction of controls (Almeida et al, 2013). Taken together with the literature, our study suggests a therapeutic benefit of CBD on social interaction deficits in poly I:C rat offspring and aligns with the beneficial effects seen in schizophrenia patients and most preclinical models. Additional studies investigating other negative symptoms, such as anhedonia and avolition (assessed in rodents using intra-cranial self-stimulation) (Carlezon and Chartoff, 2007), would be useful to further confirm the therapeutic benefit of $\mathrm{CBD}$ on negative schizophrenia-like phenotypes.

The findings of the present study align with a growing body of evidence, suggesting beneficial effects of CBD on cognitive deficits induced by numerous pharmacological and pathological factors (reviewed in Osborne et al (2017)). Preclinical studies show a therapeutic benefit of CBD on recognition memory deficits in rodent models of Alzheimer's disease (Cheng et al, 2014a; Fagherazzi et al, 2011) and cerebral malaria (Campos et al, 2015), as well as working memory deficits induced by hepatic encephalopathy (Magen et al, 2009). In the present study, CBD treatment improved the working memory deficits of poly I:C offspring in the T-maze test. This is the first study to investigate the effect of $\mathrm{CBD}$ on working memory deficits in a preclinical model of schizophrenia phenotypes. The deficits caused by poly I:C coincide with Connor et al (2012), who reported a reduced number of correct alternations in the $\mathrm{T}$-maze test of offspring exposed to poly I:C during late gestation, indicating impaired working memory. In the present study, the latency to first entry did not differ between treatment groups in the T-maze test. The delay to first entry can be influenced by general motivation towards an incentive, arousal and attention (Pioli et al, 2014), providing an overall indication of the rodent's motivation to explore its environment and complete the alternation task (Deacon and Rawlins, 2006). Therefore, in the present study, the lower percentage of correct responses in the poly I:C group, coupled with no change in the latency to first entry in the present study, suggests that the outcome is due to working memory impairment and was not influenced by internal processes such as motivation.

This is the first study to investigate the effects of CBD treatment on recognition memory in a prenatal poly I:C infection model. The only other study to investigate the effect of $\mathrm{CBD}$ on recognition memory in schizophrenia reported that chronic, high-dose CBD treatment $(60 \mathrm{mg} / \mathrm{kg})$ attenuated object recognition memory deficits induced by MK-801 administration (Gomes et al, 2015a,b). MK-801 is a $\mathrm{N}$-methyl-D-aspartate (NMDA) receptor antagonist that is used to model aspects of schizophrenia but, unlike maternal poly I:C exposure, it does not mimic the developmental aspects of the disease (Mouri et al, 2013). Recognition memory impairments have been reported previously in the poly I:C model; however, the dose, frequency, and timing of poly I:C administration, as well as the age of the rodents during behavioral testing (adolescent $v s$ adulthood) vary across studies (Ibi et al, 2009; Li et al, 2014; Ozawa et al, 2006; Ratnayake et al, 2014; Wolff et al, 2011). On the other hand, a previous study using a similar paradigm $(4 \mathrm{mg} / \mathrm{kg}$ poly I:C, administered at GD17) reported no recognition memory deficits in adult male or female rat offspring following exposure to poly I:C in late gestation (Howland et al, 2012). This contrast may be due to differences in the NOR test protocols used, as the delay between the familiarization and test trials was $24 \mathrm{~h}$ (Howland et al, 2012), compared to the $1 \mathrm{~h}$ delay in the present study. The extended delay may give rise to examination of long term memory as well (Antunes and Biala, 2012), whereas the present study design aimed to assess recognition memory using a shorter inter-trial delay. In the present study, total object exploration times did not differ between the treatment groups. Taken together, this suggests that the lower discrimination ratio observed in poly I:C offspring was not due to lack of object exploration (ie impaired motor activity) or an aversion to novel stimuli (ie a tendency to spend more time with the familiar object indicated by a discrimination ratio closer to 0) (as described in Deacon and Rawlins (2006)), but a lack of discrimination between the familiar and novel objects.

The effects of current APDs on social interaction, recognition and working memory have been reported previously in preclinical models. Studies examining social behavioral deficits have shown an improvement (Calzavara et al, 2011; Deiana et al, 2015; Gururajan et al, 2012; Hołuj et al, 2015; Kamińska and Rogóż, 2015) or no change (Deiana et al, 2015; Gao and Li, 2014; Hołuj et al, 2015; Snigdha and Neill, 2008) following administration with olanzapine, risperidone, and aripiprazole. In addition, adolescent olanzapine administration impaired working memory of adult rats in the T-maze test (Milstein et al, 2013), while olanzapine and risperidone administration improved MK-801- and PCP-induced object recognition memory deficits in mice and rats (Li et al, 2016; Mutlu et al, 2011; Snigdha et al, 2010). Recognition and working memory impairments have been linked to dysfunction in the dopaminergic and glutamatergic/GABAergic systems, particularly in the hippocampus and cortical circuitry (AbiDargham and Moore, 2003; Antunes and Biala, 2012), and these systems are highly implicated in schizophrenia pathology (reviewed in Lewis (2012)). Hyperfunction in the dopaminergic mesolimbic pathway, which projects from the ventral tegmental area (VTA) to the nucleus accumbens (NAc), is implicated in the positive symptoms of schizophrenia (Brisch et al, 2014). Conversely, hypofunction in the dopaminergic mesocortical pathway, which projects from the VTA to the prefrontal cortex (PFC), is implicated in the negative and cognitive symptoms of schizophrenia (Goldman-Rakic et al, 2004). Dopamine also modulates glutamergic activity directly through dopamine D1 receptors 
located on pyramidal neurons or indirectly through dopamine D1/D2R co-localization on GABAergic interneurons, indicating an interaction between the dopaminergic and glutamatergic/GABAergic systems in schizophrenia (Laruelle, 2014). Interestingly, poly I:C offspring also exhibit altered dopaminergic signaling (Luchicchi et al, 2016; Meyer et al, 2008) and decreased expression of glutamatergic (eg NR1 subunit of the NMDAR) and GABAergic markers (eg $\mathrm{GAD}_{67}$ and the calcium-binding protein parvalbumin) in the PFC and hippocampus (Forrest et al, 2012; Ibi et al, 2009; Meyer et al, 2008), reflecting some of the molecular changes observed in schizophrenia patients.

There is limited literature investigating the mechanisms underlying the therapeutic benefits of CBD in neuropsychiatric disorders (Osborne et al, 2017); however, recent studies have suggested that $\mathrm{CBD}$ interacts with the dopaminergic system (Renard et al, 2016; Seeman, 2016). For example, CBD inhibited binding of the selective dopamine D2R antagonist, domperidone, in rat striatal tissue in the same manner as the APD, aripiprazole. In contrast to other atypical APDs (such as olanzapine) that antagonize the D2R, CBD is hypothesized to act as a partial agonist at D2Rs to normalize dopaminergic signaling (Seeman, 2016). In addition, CBD administered into the NAc attenuated amphetamine-induced dopaminergic hyperfunction (via the mammalian target of rapamycin (mTOR) signaling) in the VTA of rats (Renard et al, 2016). Given that CBD attenuates dopaminergic hyperfunction in the mesolimbic pathway and shows partial agonistic activity at D2Rs, it is possible that CBD may also normalize dopaminergic hypofunction in the mesocortical pathway to improve negative and cognitive symptoms of schizophrenia. Limited evidence suggests that CBD also interacts with the glutamatergic and GABAergic systems. For example, CBD restored MK-801-induced deficits in parvalbumin expression and GRIN1 mRNA expression (gene that encodes for the NR1 subunit of the NMDAR) in the PFC and hippocampus, respectively (Gomes et al, 2015a). Further investigation into the impact of CBD on these major neurotransmitter systems implicated in schizophrenia pathology may shed light on the mechanisms underlying the improvement of social behavior, recognition, and working memory in poly I:C offspring in the present study.

It is important to note that while CBD improved social interaction, working and recognition memory deficits in poly I:C offspring in the present study, it had no effect in control offspring. This observation is supported by previous preclinical studies (Barichello et al, 2012; Campos et al, 2015; Cassol-Jr et al, 2010; Cheng et al, 2014a, 2014b; Fagherazzi et al, 2011; Long et al, 2012) and demonstrates a tendency for $\mathrm{CBD}$ to improve deficits associated with pathological states, rather than alter behavior in healthy rodents. In the present study, CBD was administered twicedaily based on evidence that the half-life of the drug is $\sim 11 \mathrm{~h}$ in the rat brain following an intraperitoneal injection (Deiana et al, 2011). The dose of CBD used in the present study $(10 \mathrm{mg} / \mathrm{kg})$ was based on previous studies that reported improvement of cognitive impairment in rodent models, without negative side-effects (Barichello et al, 2012; Cassol-Jr et al, 2010; Fagherazzi et al, 2011; Schiavon et al, 2014). Consideration of the biphasic dose-response of CBD is important as high doses of CBD can lead to negative effects such as sedation $(600 \mathrm{mg})$ and hyperprolactinemia $(120-240 \mathrm{mg} / \mathrm{kg})$ in healthy humans and rats, respectively, which are the side-effects also associated with APDs (Zuardi, 2008). Further research into any long-term negative effects of CBD would be prudent. Current second generation APDs such as olanzapine, clozapine, and risperidone are also associated with metabolic side effects, including diabetes and obesity that can hinder the treatment of schizophrenia patients (Weston-Green et al, 2013). We found no effect of CBD on total body weight-gain, food intake or water intake over the treatment period. Using a female rodent model, our laboratory has shown that APD administration increases body weight-gain and food intake, and is associated with changes in metabolic signaling pathways in the brain and periphery (Weston-Green et al, 2011, 2012a, 2012b). In humans, women are particularly sensitive to the metabolic side effects of APDs (Seeman, 2009) and female rats mimic this sensitivity (Weston-Green et al, 2010), while male rats appear to confer a level of resistance to APD-induced obesity (Minet-Ringuet et al, 2006; Weston-Green et al, 2010) but the mechanisms are unclear. A limitation of the present study is the use of males only; further studies investigating the effects of CBD on metabolic parameters, as well as CBD's ability to restore social and cognitive poly I:C-induced deficits in female rats are necessary. Nevertheless, the lack of weight-gain change in CBD-treated poly I:C offspring aligns with findings from a clinical trial that reported no alterations in the body weight of schizophrenia patients ( $82 \%$ male), following one month of CBD treatment (Leweke et al, 2012). Overall, our finding adds to the limited literature suggesting that CBD may have low weight-gain liability. The present study did not employ a cross-fostering paradigm. Previous studies have shown that some behavioral deficits (eg latent inhibition and conditioned fear) are present in control offspring cross-fostered to poly I:C dams (Meyer et al, 2006; Schwendener et al, 2008), suggesting that changes in maternal behaviour can influence the behavioral phenotype of offspring. Future studies could address the potential influence of maternal behavioral changes on the phenotypes observed in the present study.

\section{Summary}

This study has shown for the first time that CBD can improve recognition and working memory and social interaction deficits in a prenatal poly I:C infection model, reinforcing the potential of $\mathrm{CBD}$ to ameliorate the negative symptoms of schizophrenia. Chronic CBD treatment increased preference for the novel object in the NOR, improved working memory performance in the T-maze test and restored social interaction deficits in poly I:C offspring. Future studies investigating the efficacy of CBD to improve cognition in this model would benefit from considering additional cognitive domains that are impaired in neuropsychiatric disorders, including spatial learning and memory, attention or cognitive flexibility as outcome measures. Although the mechanisms of action of CBD are not well understood, $\mathrm{CBD}$ may exert its therapeutic effects on cognition by targeting key neurotransmitter systems (eg dopaminergic, glutamatergic, GABAergic) implicated in cognitive function, but more research is needed to elucidate the underlying mechanisms. Nevertheless, these novel 
findings present interesting implications for the use of $\mathrm{CBD}$ to treat the social and cognitive deficits associated with disorders such as schizophrenia.

\section{FUNDING AND DISCLOSURE}

This study was supported by the University of Wollongong utilizing funding from a Faculty of Science, Medicine and Health Advancement Grant (2015/SPGA-S/02) awarded to KWG and XFH; this source had no further role in the study design, decision to publish, or preparation of the manuscript. ALO is supported by an Australian Government Research Training Program Scholarship from the University of Wollongong. NS was supported by an Australian Research Council Future Fellowship (FT110100752). IB is supported by a Postgraduate Research Scholarship from the University of Wollongong and Illawarra Shoalhaven Local Health District. The authors declare no conflict of interest.

\section{ACKNOWLEDGMENTS}

We thank Cree de Clouett, Martin Engel, Ashleigh Gorak, Paul Le Mesurier, Luisa Lufe, Nicole Miles, Jessica Nealon, Anita-Louise Obeid, Tereza Pejovska, Dominic Sellers, and Erika Svenson for their technical assistance during the animal experiments.

\section{REFERENCES}

Abi-Dargham A, Moore H (2003). Prefrontal DA transmission at D1 receptors and the pathology of schizophrenia. Neuroscientist 9: 404-416.

Almeida V, Levin R, Peres FF, Niigaki ST, Calzavara MB, Zuardi AW et al (2013). Cannabidiol exhibits anxiolytic but not antipsychotic property evaluated in the social interaction test. Prog Neuropsychopharmacol Biol Psychiatry 41: 30-35.

Andersen SL, Navalta CP (2004). Altering the course of neurodevelopment: a framework for understanding the enduring effects of psychotropic drugs. Int J Dev Neurosci 22: 423-440.

Antunes M, Biala G (2012). The novel object recognition memory: neurobiology, test procedure, and its modifications. Cogn Process 13: 93-110.

Barch DM, Ceaser A (2012). Cognition in schizophrenia: core psychological and neural mechanisms. Trends Cogn Sci 16: 27-34.

Barichello T, Ceretta RA, Generoso JS, Moreira AP, Simões LR, Comim CM et al (2012). Cannabidiol reduces host immune response and prevents cognitive impairments in Wistar rats submitted to pneumococcal meningitis. Eur J Pharmacol 697: 158-164.

Bevins RA, Besheer J (2006). Object recognition in rats and mice: a one-trial non-matching-to-sample learning task to study 'recognition memory'. Nat Protoc 1: 1306-1311.

Brisch R, Saniotis A, Wolf R, Bielau H, Bernstein H-G, Steiner J et al (2014). The role of dopamine in schizophrenia from a neurobiological and evolutionary perspective: old fashioned, but still in vogue. Schizophrenia 5: 1-11.

Buschert J, Sakalem ME, Saffari R, Hohoff C, Rothermundt M, Arolt V et al (2016). Prenatal immune activation in mice blocks the effects of environmental enrichment on exploratory behavior and microglia density. Prog Neuropsychopharmacol Biol Psychiatry 67: 10-20.

Calzavara MB, Levin R, Medrano WA, Almeida V, Sampaio APF, Barone LC et al (2011). Effects of antipsychotics and amphetamine on social behaviors in spontaneously hypertensive rats. Behav Brain Res 225: 15-22.

Campos AC, Brant F, Miranda AS, Machado FS, Teixeira AL (2015). Cannabidiol increases survival and promotes rescue of cognitive function in a murine model of cerebral malaria. Neuroscience 289: 166-180.

Careaga M, Murai T, Bauman MD (2016). Maternal immune activation and autism spectrum disorder: From rodents to nonhuman and human primates. Biol Psychiatry 81: 391-401.

Carlezon WA, Chartoff EH (2007). Intracranial self-stimulation (ICSS) in rodents to study the neurobiology of motivation. Nat Protoc 2: 2987-2995.

Cassol-Jr OJ, Comim CM, Silva BR, Hermani FV, Constantino LS, Felisberto F et al (2010). Treatment with cannabidiol reverses oxidative stress parameters, cognitive impairment and mortality in rats submitted to sepsis by cecal ligation and puncture. Brain Res 1348: 128-138.

Cheng D, Low JK, Logge W, Garner B, Karl T (2014a). Chronic cannabidiol treatment improves social and object recognition in double transgenic APPswe/PS1 $\mathrm{E} 9$ mice. Psychopharmacology 231: 3009-3017.

Cheng D, Spiro AS, Jenner AM, Garner B, Karl T (2014b). Longterm cannabidiol treatment prevents the development of social recognition memory deficits in Alzheimer's disease transgenic mice. J Alzheimers Dis 42: 1383-1396.

Connor CM, Dincer A, Straubhaar J, Galler JR, Houston IB, Akbarian S (2012). Maternal immune activation alters behavior in adult offspring, with subtle changes in the cortical transcriptome and epigenome. Schizophr Res 140: 175-184.

Deacon RMJ, Rawlins JNP (2006). T-maze alternation in the rodent. Nat Protoc 1: 7-12.

De Santis M, Lian J, Huang X-F, Deng C (2016). Early antipsychotic treatment in childhood/adolescent period has long-term effects on depressive-like, anxiety-like and locomotor behaviours in adult rats. J Psychopharmacol 30: 204-214.

Deiana S, Watanabe A, Yamasaki Y, Amada N, Arthur M, Fleming S et al (2011). Plasma and brain pharmacokinetic profile of cannabidiol (CBD), cannabidivarine (CBDV), $\Delta$ 9-tetrahydrocannabivarin (THCV) and cannabigerol (CBG) in rats and mice following oral and intraperitoneal administration and CBD action on obsessivecompulsive behaviour. Psychopharmacology 219: 859-873.

Deiana S, Watanabe A, Yamasaki Y, Amada N, Kikuchi T, Stott C et al (2015). MK-801-induced deficits in social recognition in rats: reversal by aripiprazole, but not olanzapine, risperidone, or cannabidiol. Behav Pharmacol 26: 748-765.

Du Bois TM, Huang X-F, Deng C (2008). Perinatal administration of PCP alters adult behaviour in female Sprague-Dawley rats. Behav Brain Res 188: 416-419.

Fagherazzi EV, Garcia VA, Maurmann N, Bervanger T, Halmenschlager LH, Busato SB et al (2011). Memory-rescuing effects of cannabidiol in an animal model of cognitive impairment relevant to neurodegenerative disorders. Psychopharmacology 219: 1133-1140.

Forrest CM, Khalil OS, Pisar M, Smith RA, Darlington L, Stone TW (2012). Prenatal activation of Toll-like receptors-3 by administration of the viral mimetic poly(I:C) changes synaptic proteins, $\mathrm{N}$-methyl-D-aspartate receptors and neurogenesis markers in offspring. Mol Brain 5: 22.

Gao J, Li M (2014). Differential effects of intermittent versus continuous haloperidol treatment throughout adolescence on haloperidol sensitization and social behavior in adulthood. Prog Neuropsychopharmacol Biol Psychiatry 54: 67-75.

Goldman-Rakic PS, Castner SA, Svensson TH, Siever LJ, Williams GV (2004). Targeting the dopamine D1 receptor in schizophrenia: insights for cognitive dysfunction. Psychopharmacology 174: 3-16.

Gomes FV, Issy AC, Ferreira FR, Viveros M-P, Del Bel EA, Guimaraes FS (2015a). Cannabidiol attenuates sensorimotor 
gating disruption and molecular changes induced by chronic antagonism of NMDA receptors in mice. Int J Neuropsychopharmacol 18: 1-10.

Gomes FV, Llorente R, Del Bel EA, Viveros M-P, López-Gallardo M, Guimarães FS (2015b). Decreased glial reactivity could be involved in the antipsychotic-like effect of cannabidiol. Schizophr Res 164: 155-163.

Gray JA, Roth BL (2007). Molecular targets for treating cognitive dysfunction in schizophrenia. Schizophr Bull 33: 1100-1119.

Green MF (2006). Cognitive impairment and functional outcome in schizophrenia and bipolar disorder. J Clin Psychiatry 67: e12.

Gururajan A, Taylor DA, Malone DT (2011). Effect of cannabidiol in a MK-801-rodent model of aspects of Schizophrenia. Behav Brain Res 222: 299-308.

Gururajan A, Taylor DA, Malone DT (2012). Cannabidiol and clozapine reverse MK-801-induced deficits in social interaction and hyperactivity in Sprague-Dawley rats. J Psychopharmacol (Oxf) 26: 1317-1332.

Hallak JEC, Machado-de-Sousa JP, Crippa JAS, Sanches RF, Trzesniak C, Chaves C et al (2010). Performance of schizophrenic patients in the Stroop Color Word Test and electrodermal responsiveness after acute administration of cannabidiol (CBD). Rev Bras Psiquiatr 32: 56-61.

Hołuj M, Popik P, Nikiforuk A (2015). Improvement of ketamineinduced social withdrawal in rats: the role of 5-HT7 receptors. Behav Pharmacol 26: 766-775.

Howland JG, Cazakoff BN, Zhang Y (2012). Altered object-in-place recognition memory, prepulse inhibition, and locomotor activity in the offspring of rats exposed to a viral mimetic during pregnancy. Neuroscience 201: 184-198.

Ibi D, Nagai T, Kitahara Y, Mizoguchi H, Koike H, Shiraki A et al (2009). Neonatal polyI:C treatment in mice results in schizophrenia-like behavioral and neurochemical abnormalities in adulthood. Neurosci Res 64: 297-305.

Kamińska K, Rogóż Z (2015). The effect of combined treatment with risperidone and antidepressants on the MK-801-induced deficits in the social interaction test in rats. Pharmacol Rep 67: 1183-1187.

Kilkenny C, Browne WJ, Cuthill IC, Emerson M, Altman DG (2010). Improving bioscience research reporting: the ARRIVE guidelines for reporting animal research. PLoS Biol 8: 1-5.

Labouesse MA, Dong E, Grayson DR, Guidotti A, Meyer U (2015). Maternal immune activation induces GAD1 and GAD2 promoter remodeling in the offspring prefrontal cortex. Epigenetics 10: 1143-1155.

Laruelle M (2014). Schizophrenia: from dopaminergic to glutamatergic interventions. Curr Opin Pharmacol 14: 97-102.

Leweke FM, Piomelli D, Pahlisch F, Muhl D, Gerth CW, Hoyer C et al (2012). Cannabidiol enhances anandamide signaling and alleviates psychotic symptoms of schizophrenia. Transl Psychiatry 2: e94.

Lewis DA (2012). Cortical circuit dysfunction and cognitive deficits in schizophrenia: Implications for preemptive interventions. Eur J Neurosci 35: 1871-1878.

Li C, Tang Y, Yang J, Zhang X, Liu Y, Tang A (2016). Sub-chronic antipsychotic drug administration reverses the expression of neuregulin 1 and ErbB4 in a cultured MK801-induced mouse primary hippocampal neuron or a neurodevelopmental schizophrenia model. Neurochem Res 41: 2049-2064.

Li W, Chang Y, Lee LJH, Lee L (2014). Prenatal infection affects the neuronal architecture and cognitive function in adult mice. Dev Neurosci 36: 359-370.

Lindenmayer J-P, Nasrallah H, Pucci M, James S, Citrome L (2013). A systematic review of psychostimulant treatment of negative symptoms of schizophrenia: challenges and therapeutic opportunities. Schizophr Res 147: 241-252.

Long LE, Chesworth R, Huang X-F, Wong A, Spiro A, McGregor IS et al (2012). Distinct neurobehavioural effects of cannabidiol in transmembrane domain neuregulin 1 mutant mice. PLoS ONE 7: e34129.

Luchicchi A, Lecca S, Melis M, De Felice M, Cadeddu F, Frau R et al (2016). Maternal immune activation disrupts dopamine system in the offspring. Int J Neuropsychopharmacol 19: 1-10.

Magen I, Avraham Y, Ackerman Z, Vorobiev L, Mechoulam R, Berry EM (2009). Cannabidiol ameliorates cognitive and motor impairments in mice with bile duct ligation. J Hepatol 51: 528-534.

Meyer U (2013). Developmental neuroinflammation and schizophrenia. Prog Neuropsychopharmacol Biol Psychiatry 42: 20-34.

Meyer U (2014). Prenatal poly(I:C) exposure and other developmental immune activation models in rodent systems. Biol Psychiatry 75: 307-315.

Meyer U, Feldon J (2012). To poly(I:C) or not to poly(I:C): Advancing preclinical schizophrenia research through the use of prenatal immune activation models. Neuropharmacology 62: 1308-1321.

Meyer U, Feldon J, Dammann O (2011a). Schizophrenia and autism: both shared and disorder-specific pathogenesis via perinatal inflammation? Pediatr Res 69: 26R-33R.

Meyer U, Feldon J, Fatemi SH (2009). In-vivo rodent models for the experimental investigation of prenatal immune activation effects in neurodevelopmental brain disorders. Neurosci Biobehav Rev 33: 1061-1079.

Meyer U, Nyffeler M, Yee BK, Knuesel I, Feldon J (2008). Adult brain and behavioral pathological markers of prenatal immune challenge during early/middle and late fetal development in mice. Brain Behav Immun 22: 469-486.

Meyer U, Schwarz MJ, Müller N (2011b). Inflammatory processes in schizophrenia: A promising neuroimmunological target for the treatment of negative/cognitive symptoms and beyond. Pharmacol Ther 132: 96-110.

Meyer U, Schwendener S, Feldon J, Yee BK (2006). Prenatal and postnatal maternal contributions in the infection model of schizophrenia. Exp Brain Res 173: 243-257.

Milstein JA, Elnabawi A, Vinish M, Swanson T, Enos JK, Bailey AM et al (2013). Olanzapine treatment of adolescent rats causes enduring specific memory impairments and alters cortical development and function. PLOS ONE 8: 1-17.

Minet-Ringuet J, Even PC, Goubern M, Tomé D, de Beaurepaire R (2006). Long term treatment with olanzapine mixed with the food in male rats induces body fat deposition with no increase in body weight and no thermogenic alteration. Appetite 46: 254-262.

Mouri A, Nagai T, Ibi D, Yamada K (2013). Animal models of schizophrenia for molecular and pharmacological intervention and potential candidate molecules. Neurobiol Dis 53: 61-74.

Mutlu O, Ulak G, Celikyurt IK, Akar FY, Erden F, Tanyeri P (2011). Effects of olanzapine, sertindole and clozapine on MK-801 induced visual memory deficits in mice. Pharmacol Biochem Behav 99: 557-565.

NHMRC (2013). Australian Code of Practice for the Care and Use of Animals for Scientific Purposes 8th edition. Australian Government Canberra. https://www.nhmrc.gov.au/_files_nhmrc/ publications/attachments/ea28_code_care_use_animals_131209. pdf.

Osborne AL, Solowij N, Weston-Green K (2017). A systematic review of the effect of cannabidiol on cognitive function: Relevance to schizophrenia. Neurosci Biobehav Rev 72: 310-324.

Ozawa K, Hashimoto K, Kishimoto T, Shimizu E, Ishikura H, Iyo M (2006). Immune activation during pregnancy in mice leads to dopaminergic hyperfunction and cognitive impairment in the offspring: a neurodevelopmental animal model of schizophrenia. Biol Psychiatry 59: 546-554.

Peres FF, Diana MC, Suiama MA, Justi V, Almeida V, Bressan RA et al (2016). Peripubertal treatment with cannabidiol prevents the emergence of psychosis in an animal model of schizophrenia. Schizophr Res 172: 220-221. 
Pioli EY, Gaskill BN, Gilmour G, Tricklebank MD, Dix SL, Bannerman D et al (2014). An automated maze task for assessing hippocampussensitive memory in mice. Behav Brain Res 261: 249-257.

Piontkewitz Y, Arad M, Weiner I (2011). Risperidone administered during asymptomatic period of adolescence prevents the emergence of brain structural pathology and behavioral abnormalities in an animal model of schizophrenia. Schizophr Bull 37: 1257-1269.

Piontkewitz Y, Assaf Y, Weiner I (2009). Clozapine administration in adolescence prevents postpubertal emergence of brain structural pathology in an animal model of schizophrenia. Biol Psychiatry 66: 1038-1046.

Ratnayake U, Quinn T, Larosa DA, Dickinson H, Walker DW (2014). Prenatal exposure to the viral mimetic poly I:C alters fetal brain cytokine expression and postnatal behaviour. Dev Neurosci 36: 83-94.

Renard J, Loureiro M, Rosen LG, Zunder J, de Oliveira C, Schmid S et al (2016). Cannabidiol counteracts amphetamine-induced neuronal and behavioral sensitization of the mesolimbic dopamine pathway through a novel mTOR/p70S6 kinase signaling pathway. J Neurosci 36: 5160-5169.

Richetto J, Labouesse MA, Poe MM, Cook JM, Grace AA, Riva MA et al (2015). Behavioral effects of the benzodiazepine-positive allosteric modulator $\mathrm{SH}-053-2$ ' $\mathrm{F}-\mathrm{S}-\mathrm{CH}_{3}$ in an immune-mediated neurodevelopmental disruption model. Int J Neuropsychopharmacol 18: 1-11.

Schiavon AP, Soares LM, Bonato JM, Milani H, Guimarães FS, de Oliveira RMW (2014). Protective effects of cannabidiol against hippocampal cell death and cognitive impairment induced by bilateral common carotid artery occlusion in mice. Neurotox Res 26: 307-316.

Schubart CD, Sommer IEC, Fusar-Poli P, de Witte L, Kahn RS, Boks MPM (2014). Cannabidiol as a potential treatment for psychosis. Eur Neuropsychopharmacol 24: 51-64.

Schwendener S, Meyer U, Feldon J (2008). Deficient maternal care resulting from immunological stress during pregnancy is associated with a sex-dependent enhancement of conditioned fear in the offspring. J Neurodev Disord 1: 15.

Seeman MV (2009). Secondary effects of antipsychotics: women at greater risk than men. Schizophr Bull 35: 937-948.
Seeman P (2016). Cannabidiol is a partial agonist at dopamine D2High receptors, predicting its antipsychotic clinical dose. Transl Psychiatry 6: e920.

Snigdha S, Horiguchi M, Huang M, Li Z, Shahid M, Neill JC et al (2010). Attenuation of phencyclidine-induced object recognition deficits by the combination of atypical antipsychotic drugs and pimavanserin (ACP 103), a 5-hydroxytryptamine(2A) receptor inverse agonist. J Pharmacol Exp Ther 332: 622-631.

Snigdha S, Neill JC (2008). Efficacy of antipsychotics to reverse phencyclidine-induced social interaction deficits in female rats-a preliminary investigation. Behav Brain Res 187: 489-494.

Weston-Green K, Huang X-F, Deng C (2010). Sensitivity of the female rat to olanzapine-induced weight gain-far from the clinic? Schizophr Res 116: 299-300.

Weston-Green K, Huang X-F, Deng C (2011). Olanzapine treatment and metabolic dysfunction: a dose response study in female Sprague Dawley rats. Behav Brain Res 217: 337-346.

Weston-Green K, Huang X-F, Deng C (2012a). Alterations to melanocortinergic, gABAergic and cannabinoid neurotransmission associated with olanzapine-induced weight gain. PLoS ONE 7: 1-12.

Weston-Green K, Huang X-F, Deng C (2013). Second generation antipsychotic-induced type 2 diabetes: a role for the muscarinic M3 receptor. CNS Drugs 27: 1069-1080.

Weston-Green K, Huang X-F, Lian J, Deng C (2012b). Effects of olanzapine on muscarinic M3 receptor binding density in the brain relates to weight gain, plasma insulin and metabolic hormone levels. Eur Neuropsychopharmacol 22: 364-373.

Wilson CA, Koenig JI (2014). Social interaction and social withdrawal in rodents as readouts for investigating the negative symptoms of schizophrenia. Eur Neuropsychopharmacol 24: 759-773.

Wolff AR, Cheyne KR, Bilkey DK (2011). Behavioural deficits associated with maternal immune activation in the rat model of schizophrenia. Behav Brain Res 225: 382-387.

Zhu F, Zheng Y, Liu Y, Zhang X, Zhao J (2014). Minocycline alleviates behavioral deficits and inhibits microglial activation in the offspring of pregnant mice after administration of polyriboinosinic-polyribocytidilic acid. Psychiatry Res 219: 680-686.

Zuardi AW (2008). Cannabidiol: from an inactive cannabinoid to a drug with wide spectrum of action. Rev Bras Psiquiatr 30: 271-280.

Supplementary Information accompanies the paper on the Neuropsychopharmacology website (http://www.nature.com/npp) 